\title{
Implementation of synthetic aperture imaging on a hand-held device
}

Hemmsen, Martin Christian; Kjeldsen, Thomas; Larsen, Lee; Kjær, Carsten; Tomov, Borislav Gueorguiev; Mosegaard, Jesper; Jensen, Jørgen Arendt

\section{Published in:}

Proceedings of 2014 IEEE International Ultrasonics Symposium

Link to article, DOI:

10.1109/ULTSYM.2014.0542

Publication date:

2014

Document Version

Early version, also known as pre-print

Link back to DTU Orbit

Citation (APA):

Hemmsen, M. C., Kjeldsen, T., Larsen, L., Kjær, C., Tomov, B. G., Mosegaard, J., \& Jensen, J. A. (2014). Implementation of synthetic aperture imaging on a hand-held device. In Proceedings of 2014 IEEE International Ultrasonics Symposium (pp. 2177-2180). IEEE. https://doi.org/10.1109/ULTSYM.2014.0542

\section{General rights}

Copyright and moral rights for the publications made accessible in the public portal are retained by the authors and/or other copyright owners and it is a condition of accessing publications that users recognise and abide by the legal requirements associated with these rights.

- Users may download and print one copy of any publication from the public portal for the purpose of private study or research.

- You may not further distribute the material or use it for any profit-making activity or commercial gain

- You may freely distribute the URL identifying the publication in the public portal 
Paper presented at the 2014 IEEE International Ultrasonics Symposium:

\section{Implementation of synthetic aperture imag- ing on a hand-held device}

Martin Christian Hemmsen, Thomas Kjeldse, Lee Lassen, Carsten Kjer, Borislav G. Tomov, Jesper Mosegaard and Jørgen Arendt Jensen

Center for Fast Ultrasound Imaging,

Biomedical Engineering Group,

Department of Electrical Engineering

Ørsteds Plads Building 349,

Technical University of Denmark,

2800 Kgs. Lyngby, Denmark.

To be published in the Proceedings of 2014 IEEE International Ultrasonics Symposium. 


\title{
Implementation of synthetic aperture imaging on a hand-held device
}

\author{
Martin Christian Hemmsen ${ }^{1}$, Thomas Kjeldsen ${ }^{2}$, Lee Lassen ${ }^{2}$, Carsten $\mathrm{Kjær}^{3}$, Borislav Tomov ${ }^{1}$, \\ Jesper Mosegaard ${ }^{2}$ and Jørgen Arendt Jensen ${ }^{1}$ \\ ${ }^{1}$ Center for Fast Ultrasound Imaging Dept. of Elec. Eng. Technical University of Denmark DK-2800 Lyngby, Denmark \\ ${ }^{2}$ Computer Graphics Lab, Alexandra Institute, DK-8200 Aarhus N, Denmark \\ ${ }^{3}$ BK Medical, Mileparken 34, DK-2730 Herlev, Denmark
}

\begin{abstract}
This paper presents several implementations of Synthetic Aperture Sequential Beamforming (SASB) on commercially available hand-held devices. The implementations include real-time wireless reception of ultrasound radio frequency signals and GPU processing for B-mode imaging. The proposed implementation demonstrates that SASB can be executed in-time for real-time ultrasound imaging. The wireless communication between probe and processing device satisfies the required bandwidth for real-time data transfer with current 802.11ac technology. The implementation is evaluated using four different hand-held devices all with different chipsets and a BK Medical UltraView 800 ultrasound scanner emulating a wireless probe. The wireless transmission is benchmarked using an imaging setup consisting of 269 scan lines $x 1472$ complex samples (1.58 MB pr. frame, 16 frames per second). The measured data throughput reached an average of $28.8 \mathrm{MB} / \mathrm{s}$ using a LG G2 mobile device, which is more than the required data throughput of $25.3 \mathrm{MB} / \mathrm{s}$. Benchmarking the processing performance for B-mode imaging showed a total processing time of $18.9 \mathrm{~ms}(53 \mathrm{frames} / \mathrm{s})$, which is less than the acquisition time $(62.5 \mathrm{~ms})$.
\end{abstract}

\section{INTRODUCTION}

Ultrasound technology has become progressively more portable, and now includes hand-held devices designed to complement clinical examination[1], [2], [3]. While there have been several earlier products, they all suffered from incomplete feature sets and/or compromised imaging performance. The rapid increase in processing power and ASIC integration, coupled with the development of new battery technologies, have allowed the development of hand-held systems with imaging performance nearly equivalent to cart-based systems, and battery-powered operating times of 2 hours or more [4]. This has allowed ultrasound to find new applications not previously possible, such as in emergency medical services, primary care and developing world [5], [6], [7], [8].

Conventional ultrasound imaging techniques rely on the use of multi-element transducers, where the analog signal from each element is independently wired to the processing unit. The large number of signals is contributing to a high image quality, but comes with the cost of an expensive and fragile cable with difficulties to control infection risk. Furthermore, as the computation of the B-mode image is performed on the compact processor unit on the mobile device, users can not enjoy the fruit of the rich computational power of Hospital Information Systems. Synthetic Aperture Sequential
Beamforming (SASB) [9] is a technique that produces image quality comparable to dynamical receive focusing [10], but requires much lower data bandwidth between the probe and processing unit. It is hypothesized that the low data bandwidth requirement makes it possible to substitute the analog communication link with wireless technology standards. The objective of this work is to evaluate if modern hand-held devices such as the Samsung Galaxy TabPro [11] and LG G2 [12] have the bandwidth and processing power to receive a Wi-Fi transmitted ultrasound data set and generate images in real-time, without adding specialized hardware.

\section{MATERiAls AND Methods}

Implementation of the SASB imaging technique requires integration of a simple fixed focus beamformer in the transducer handle. The beamformed data is transferred as a single stream to the main processing unit, and the final image is created by refocusing the fixed focus scan lines. Figure 1 illustrates the system architecture.

In the initial stage the beamformer operates on the signals received by the transducer array. The emission sequence scans a focused emission across the volume being imaged. The beamformer applies delay-and-sum beamforming with a fixed delay for each transducer element to create a fixed focus scan line. The delay configuration is identical in both transmit and receive. The sample values of a single scan line can be interpreted as the response from a virtual source, emitting a spherical wave, positioned at the focal point of the scan line.

The second stage beamformer takes the output of the first stage and uses it as input. The focal point from the first stage beamformer is considered a virtual source, while the samples on the scan line are considered the signal received by a virtual receive element collocated with the virtual source. Each point in the focused image line contains information from a set of spatial positions limited by the opening angle of the virtual source. A single image point is therefore potentially represented in multiple first stage focused scan lines.

The second stage beamformer creates a set of image points by combining information from the first stage focused scan lines that contain information from the spatial position of the image point.

In this work the first stage beamformer is integrated into the probe and the transmission channel is implemented using 


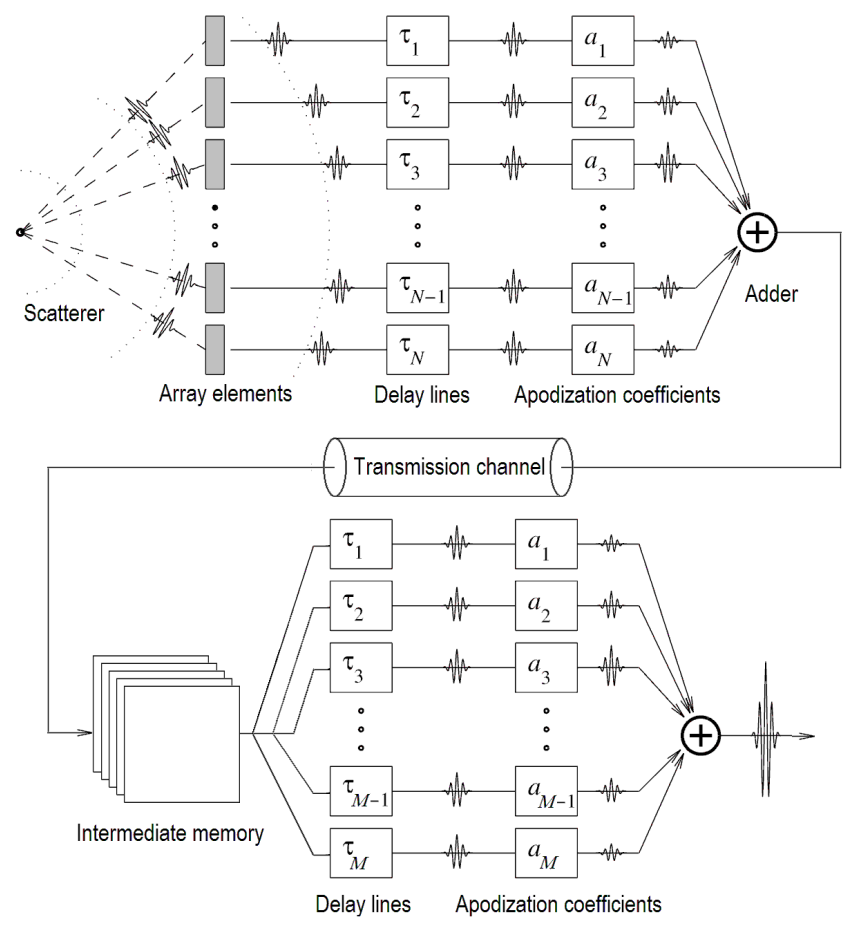

Fig. 1. System architecture. For each focused emission a simple receive beamformer create a fixed focused scan line. The scan line is transmitted ove a transmission channel and the final image is created in a second dynamic beamformer by refocusing a number of scan lines.

standard wireless 802.11ac technology. For prof-of-concept the wireless probe is emulated using a BK Medical UltraView 800 ultrasound scanner and a BK Medical 8820e convex array transducer. The scanner is configured to beamform the received echo signals using a fixed receive profile and subsequently transform data to baseband IQ data. The ultrasound scanner is programmed for a scan setup of 269 scan lines $\times 1472$ complex samples (1.58 MB pr. frame). Imaging parameters are shown in Table I.

TABLE I

IMAGING PARAMETERS

\begin{tabular}{ll}
\hline 1st stage focus depth & $70 \mathrm{~mm}$ \\
1st stage aperture size & 64 elements \\
1st stage rx-apodization & Hamming \\
1st stage tx-apodization & Box-car \\
2nd stage F\# & 2.0 \\
2nd stage apodization & Hamming \\
Excitation waveform & $3.75 \mathrm{MHz}$ 2 cycle sinusoid \\
Field of view & $60^{\circ}$ \\
Depth of view & $14.6 \mathrm{~cm}$ \\
\hline
\end{tabular}

Data is transferred using Wi-Fi from the probe to the handheld device for refocusing of the fixed focused scan lines and subsequent image processing for B-mode imaging. In order to make this second stage processing (IQ demodulation, beamforming, amplitude detection, compression and scanconversion to a screensized image) run in real-time, it is necessary to offload most computations to the GPU. Accordingly, the present work implements the aforementioned computation steps as shown in Fig. 2, where the GPU is instructed to do general purpose computations using the high level API

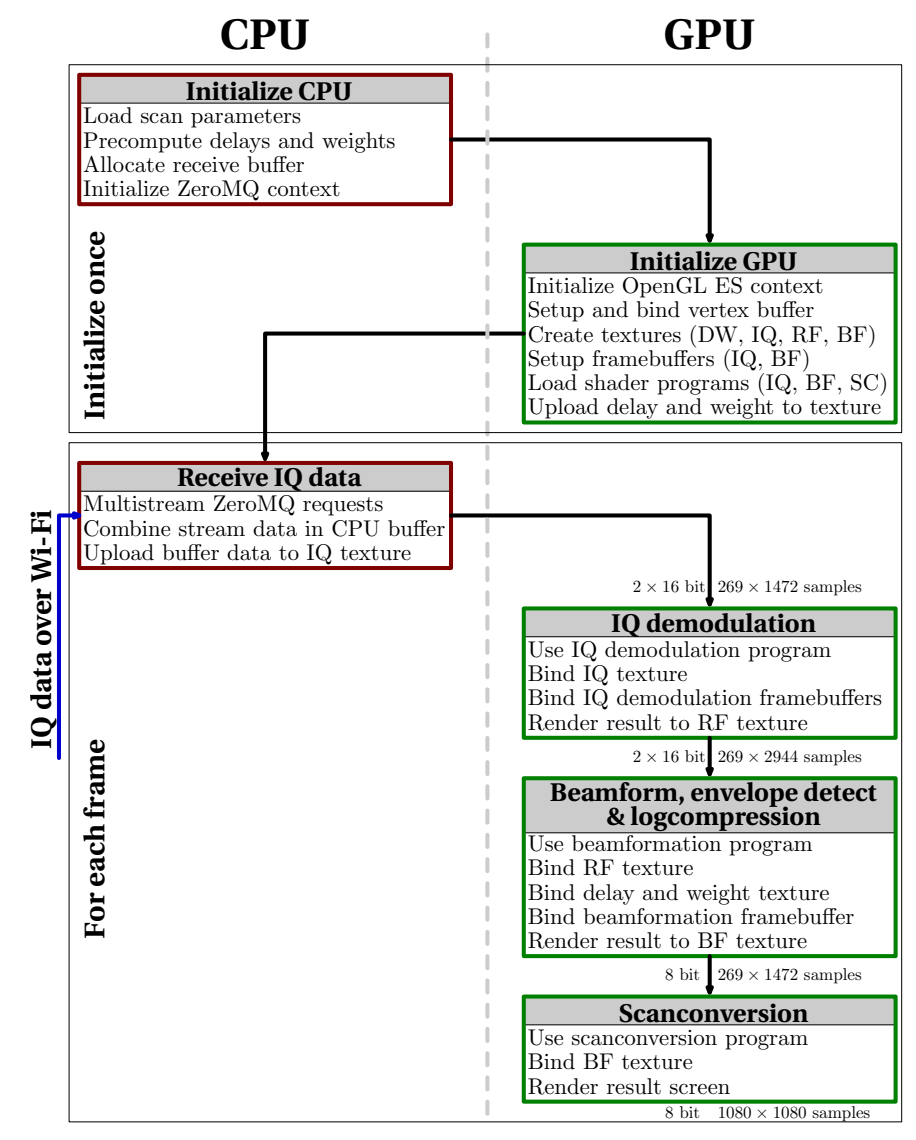

Fig. 2. Workflow diagram on the handheld device. Each of the boxes on the right illustrates one pass on the GPU.

OpenGL ES 3.0. The GPU shader code was written in GLSL ES 3.0, and the host code was written mainly in $\mathrm{C}++$ that was compiled to linux and cross-compiled to Android using the Native Development Kit. Each of the boxes on the right in Fig. 2 corresponds to a rendering pass on the GPU where computations are performed per output sample in a fragment shader. Intermediate results are stored in textures between the passes.

Many performance optimizations were considered to achieve real-time performance on low-power GPUs. All passes turn out to be memory bound, thus, an important optimization is to use 16- or 8-bit intermediate textures whenever possible. This reduces the memory bandwidth requirements to/from DRAM compared to, e.g., 32-bit single precision floating point formats. Since internal arithmetic operations within a shader program unit can still be performed with up to 32-bit precision, this data reduction turns out to be insignificant for the final image as demonstrated in Sec. III below. Another significant optimization is to precompute delays and weigths and store these values interleaved in a small 16-bit texture for optimal cache coherence.

\section{RESULTS}

The implementation is validated with a Matlab implementation on a pre-recorded data set [13]. The recorded data set is acquired using the same scan setup as the real-time implementation. Fig. 3 illustrates the generated images using (a) Matlab 
and (b) the OpenGL ES implementation. The two images are
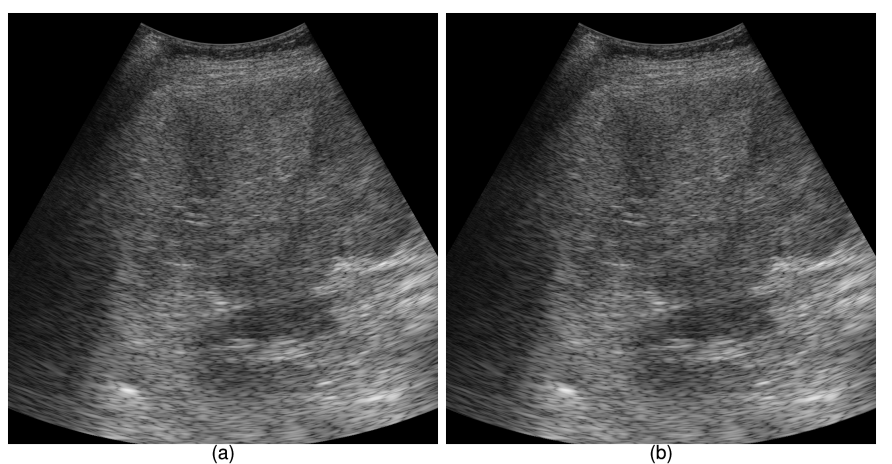

Fig. 3. In-vivo B-mode image. (a) Matlab reference implementation. (b) OpenGL ES implementation. The dynamic range is $60 \mathrm{~dB}$.

in good agreement with an average absolute pixel error of 0.41 percentage point and a root mean square error (RMSE) of 0.0087 for pixel values normalized to $[0,1]$. This corresponds to a peak signal to noise ratio $(P S N R)$ of $41.25 \mathrm{~dB}$ and a structural similarity index $(S S I M)$ of 0.9952 [14]. The small differences can be explained by the fact that Matlab uses 64-bit double precision numerical representation for all computations.

Peak signal to noise ratio is defined via the mean squared error $(M S E)$ :

$$
M S E=\frac{1}{m n} \sum_{i=0}^{m-1} \sum_{j=0}^{n-1}[I(i, j)-K(i, j)]^{2},
$$

where $K$ is a $m \times n$ image and $I$ is a reference image.

$$
P S N R=10 \log _{10}\left(\frac{M A X_{I}^{2}}{M S E}\right) .
$$

Here, $M A X_{I}$ is the maximum possible pixel value of the image.

The SSIM metric is calculated block wise of an image. The measure between two blocks $x$ and $y$ of common size is:

$$
\operatorname{SSIM}(x, y)=\frac{\left(2 \mu_{x} \mu_{y}+c_{1}\right)\left(2 \sigma_{x y}+c_{2}\right)}{\left(\mu_{x}^{2}+\mu_{y}^{2}+c_{1}\right)\left(\sigma_{x}^{2}+\sigma_{y}^{2}+c_{2}\right)},
$$

where $\mu_{x}$ is the average of $x, m u_{y}$ is the average of $y, \sigma_{x}^{2}$ is the variance of $x, \sigma_{y}^{2}$ is the variance of $y, \sigma_{x y}$ is the covariance of $x$ and $y, c_{1}=\left(k_{1} L\right)^{2}, c_{2}=\left(k_{2} L\right)^{2}$ two variables to stabilize the division with weak denominator, $L$ the dynamic range of

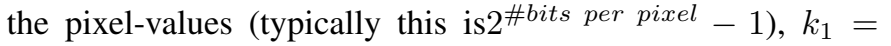
0.01 and $k_{2}=0.03$ by default.

Benchmark results were acquired for four different OpenGL ES 3.0 capable devices listed in Table II. Note that unlike

TABLE II

MOBILE DEVICES USED IN THE PRESENT BENCHMARKS.

\begin{tabular}{lllll}
\hline & LG & Samsung & Samsung & Nvidia \\
& G2 & Jalaxy Tab & Nexus 10 & Jetson TK1 \\
\hline SoC & Snapdragon 800 & Exynos 5 & Exynos 5250 & Tegra K1 \\
GPU & Adreno 330 & Mali T628 & Mali T604 & Kepler \\
Wi-Fi & 802.11ac & $802.11 \mathrm{ac}$ & $802.11 \mathrm{n}$ & $802.11 \mathrm{ac}$ \\
Screen & 1920x1080 & 2560x1600 & 2560x1600 & $1920 \times 1080$ \\
OS & Android & Android & Android & Linux4Tegra \\
\hline
\end{tabular}

the first three devices, the Jetson TK1 is not a true handheld consumer device, but a development board with similar system on chip (SoC) and GPU as very recently released tablets, such as the Nvidia Shield and the Xiaomi MiPad. Wi-Fi was added to the Jetson TK1 with an Intel 7260HMW mini PCIe expansion card.

\section{A. Evaluation of the wireless throughput}

To maximize Wi-Fi bandwidth utilization, each frame is subdivided in multiple parts, which are transferred in separate Wi-Fi streams. On the receiving side, the data from each stream is recombined in a single buffer, which is uploaded to GPU memory for further processing.

A BK Medical Ultraview 800 scanner was setup for live scanning and transmission of data using an ASUS PCEAC68 wireless network card with a theoretical speed of 1.3 Gbit/s. Real-time imaging requires $25.3 \mathrm{MB} / \mathrm{s}$ to be transferred through the transmission channel with the current imaging setup. For benchmarking purposes, however, a prerecorded dataset was emitted from a PC as fast as possible via an ASUS RT-AC68U access point in order to test the maximum transfer speed for each device. The physical setup was located in a typical office environment without any attempt to reduce surrounding wireless noise. The access point and client were separated by a few meters. The wireless throughput is measured as outgoing rate from the PC and access point, and as ingoing rate on the mobile device. Figure 4 illustrates the measured data throughtput. Each measurement is averaged over more than one minute. The figure shows that average rates

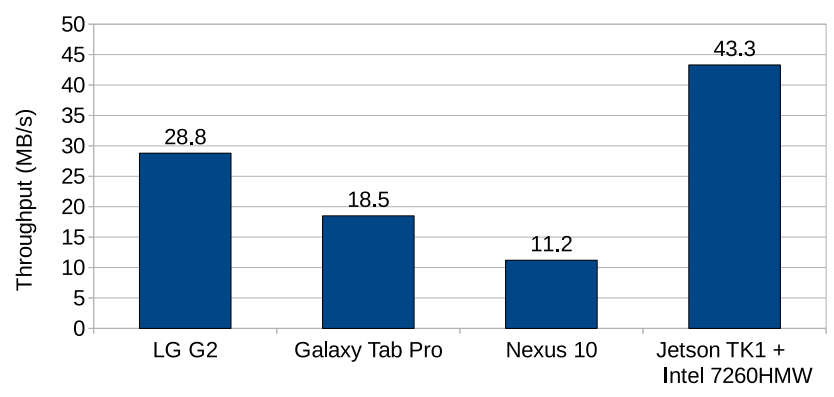

Fig. 4. Average received data rate. The Nexus 10 does not support $802.11 \mathrm{ac}$ standard and, hence, a lower throughtput is expected using the 802.11n standard.

above the real-time limit of $25.3 \mathrm{MB} / \mathrm{s}$ are indeed possible on the LG G2 and Jetson TK1.

The data transfer between the probe and the mobile device was implemented using Zero Message Queue (ØMQ). The advantage of ØMQ over standard sockets is that it allows for easy prototyping of a variety of reliable data distribution patterns and transport layer protocols. For the purpose of evaluating image quality, it was required to use a reliable transport mechanism to ensure that no data is lost in the network transfer. For future real-time applications, however, unreliable transport may also be considered, since it is often not critical that all frames are received. For example, if the Wi- 
Fi throughput is lower than the acquisition rate, it is preferable to drop frames rather than to introduce a latency.

\section{B. Evaluation of the processing performance}

The raw processing performance is benchmarked with a 24frame prerecorded dataset stored locally on the device. Thus, wireless transmission overhead is omitted from the processing measurement which only includes data upload from main memory to GPU texture memory in addition to the three GPU passes shown in Fig. 2. The OpenGL ES extension GL_EXT_disjoint_timer_query makes it possible to measure time consumptions for each of the individual GPU steps. Since this extension requires hardware and driver support beyond the OpenGL ES 3.0 standard, detailed measurements are only available on a subset of the test devices. For the other devices, only total frame timings can be achieved using the timer provided by the operating system.

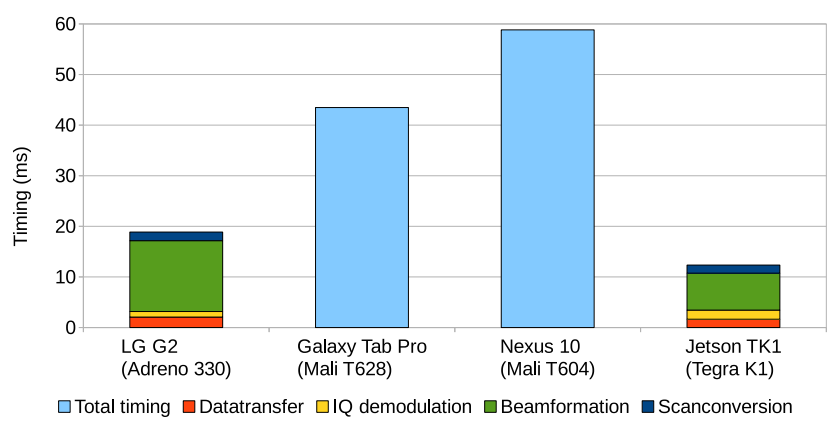

Fig. 5. Second stage processing timings. Detailed timings for the individual GPU passes were only possible for the LG G2 and Jetson TK1. The total processing time must be less than $62.5 \mathrm{~ms}$ for the current imaging setup.

Figure 5 shows that all tested devices are able to complete the whole second stage processing in less than $62.5 \mathrm{~ms}$ required for real-time performance for the current imaging parameters. In fact the fastest GPU tested is able to process each frame in just $12.3 \mathrm{~ms}$ and is thus capable of acting as a second stage beamformer for acquisition rates up to $81 \mathrm{~Hz}$.

A minor difference between the four benchmark timings is that the final image resolution is chosen to match the screensize of the individual devices. Hence, the output image is $1080 \times 1080$ for the LG G2 and the Jetson TK1 and $1600 \times 1600$ for the Galaxy Tab Pro and The Nexus 10. This difference does, however, only affect the scanconversion pass since this is the only pass renders to the onscreen framebuffer. The detailed measurements indicate that the beamformation pass is the most time consuming and that the scanconversion is only responsible for around $10 \%$ of the total timing and, thus, a larger screen resolution is only expected to introduce a minor performance penalty.

\section{DISCUSSION AND CONCLUSION}

Real-time and wireless B-mode imaging using modern hand-held devices and GPU implementations of Synthetic Aperture Sequential Beamforming (SASB) have been presented. The implementation includes wireless reception of fixed focused beamformed ultrasound signals and signal processing for synthetic aperture B-mode imaging. Performance evaluations were carried out for a typical and earlier invivo evaluated scan sequence in real-time. Evaluation of the processing power showed that current high-end mobile devices do provide sufficient processing power for high-quality realtime imaging. The measured data throughput and processing time were $28.8 \mathrm{MB} / \mathrm{s}$ and $18.9 \mathrm{~ms}$, respectively, using a LG G2 mobile device. Early tests on next-generation hardware, such as the Tegra $\mathrm{K} 1 \mathrm{SoC}$, indicate that even higher reception rates and lower processing times will be achievable in the near future.

The results show that compared to current hand-held ultrasound devices, which rely on custom made hardware, SASB can be implemented using commercially available consumer hardware. The shrinkage and cost reduction of ultrasound systems will allow ultrasound to be used in many new applications, especially rural areas with little previous access to medical imaging technologies and demand for low-cost systems. Another benefit of the SASB algorithm is the possibility to integrate the imaging mode into Hospital Information Systems. The low data bandwidth requirement makes it possible to off-load the computation to the cloud or a local cluster for advanced processing schemes.

\section{ACKNOWLEDGMENT}

This work was supported by grant 82-2012-4 from the Danish National Advanced Technology Foundation and by BK Medical.

\section{REFERENCES}

[1] GE, "GE Vscan," http://www.ge.com, 2014.

[2] Siemens, "Siemens Acuson P10," http://www.siemens.com, 2014.

[3] SonoSite, "SonoSite Nanomaxx," http://www.sonosite.com, 2014.

[4] J. Powers and F. Kremkau, "Medical ultrasound systems," Interface Focus, vol. 1, no. 4, pp. 477-489, 2011. [Online]. Available: http://rsfs.royalsocietypublishing.org/content/1/4/477.abstract

[5] B. Nelson and K. Chason, "Use of ultrasound by emergency medical services: a review," International Journal of Emergency Medicine, vol. 1, no. 4, pp. 253-259, 2008. [Online]. Available: http://dx.doi.org/10.1007/s12245-008-0075-6

[6] J. R. Immelt, V. Govindarajan, and C. Trimble, "How GE is disrupting itself," Harvard Business Review, vol. 87, no. 10, pp. 56-65, 2009.

[7] S. Sippel, K. Muruganandan, A. Levine, and S. Shah, "Review article: Use of ultrasound in the developing world," International Journal of Emergency Medicine, vol. 72, no. 4, pp. 190-197, 2011.

[8] B. P. Nelson, E. R. Melnick, and J. Li, "Portable ultrasound for remote environments, Part I: Feasibility of field deployment," The Journal of emergency medicine, vol. 40, no. 2, pp. 190-197, 2011.

[9] M. C. Hemmsen, J. M. Hansen, and J. A. Jensen, "Synthetic Aperture Sequential Beamformation applied to medical imaging using a multi element convex array transducer," in EUSAR, Apr. 2012, pp. 34-37.

[10] M. Hemmsen, P. M. Hansen, T. Lange, J. M. Hansen, K. L. Hansen, M. B. Nielsen, and J. A. Jensen, "In vivo evaluation of synthetic aperture sequential beamforming," Ultrasound Med. Biol., vol. 38, no. 4, pp. 708716, 2012.

[11] Samsung, "Samsung Galaxy TabPro," http://www.samsung.com, 2014.

[12] LG, "LG G2," http://www.lg.com, 2014.

[13] M. C. Hemmsen, S. I. Nikolov, M. M. Pedersen, M. J. Pihl, M. S Enevoldsen, J. M. Hansen, and J. A. Jensen, "Implementation of a versatile research data acquisition system using a commercially available medical ultrasound scanner," IEEE Trans. Ultrason., Ferroelec., Freq. Contr., vol. 59, no. 7, pp. 1487-1499, 2012.

[14] Z. Wang, A. Bovik, H. Sheikh, and E. Simoncelli, "Image quality assessment: from error visibility to structural similarity," Image Processing, IEEE Transactions on, vol. 13, no. 4, pp. 600-612, April 2004. 